\title{
Non-Neoplastic Lesions that may Mimic Serous Tubal Intra-Epithelial and Invasive Carcinoma of the Fallopian Tube: Morphological and Immunohistochemical Analysis
}

\author{
Giovanna Giordano* \\ Department of Medicine and Surgery Pathology Unit, University of Parma, Italy
}

Received: May 23, 2018; Published: June 05, 2018

*Corresponding author: Giovanna Giordano, Department of Medicine and Surgery Pathology Unit, University of Parma, Viale A Gramsci, 14 Parma, Italy

\begin{abstract}
In this paper the author describes the morphological and immunohistochemical features of the most common benign epithelial lesions of the salpinx, which can be mistaken for neoplastic malignancies (pseudoneoplastic lesions), on microscopic and, sometimes, also on macroscopic examination. Moreover, here it was demonstrated that both morphologic clinical data and immunohistochemical features are useful for an accurate differential pathological diagnosis from non-neoplastic lesions and serous tubal intraepithelial carcinoma (STIC) and invasive carcinoma.
\end{abstract}

Keywords: Serous Tubal Intraepithelial Carcinoma; Pseudo-Neoplastic Lesions; Malignancies; Immunohistochemical Analysis

\section{Introduction}

In this paper the author describes the morphological and immunohistochemical features of the most common benign epithelial lesions of the salpinx, which can be mistaken for neoplastic malignancies (pseudo-neoplastic lesions), on microscopic and, sometimes, also on macroscopic examination.

\section{Materials and Methods}

The material used for this study was obtained from the archive of the Department of Medicine and Surgery, Pathology Unit, Parma University (Italy) and from a review of the literature. Relevant articles were obtained by searching the PubMed and MEDLINE databases. These databases were searched using the following key words: 'Pseudoneoplastic lesions of salpinx' and 'Mimics of malignancies of salpinx. Articles were considered if the studies included clinicopathological and immunohistochemical features of the lesions. Papers, which were not written in English, were excluded.

\section{Main Text}

The main lesions that can mimic malignant neoplasms in the salpinx are a hyperplastic proliferative process of its epithelium, which may be confused with serous tubal intra-epithelial carcinoma (STIC) or invasive serous carcinoma and transitional cell metaplasia of fallopian tube fimbriae. Many of proliferative lesions can be observed in association with estrogen administration, ovar ian-produced estrogens neoplasms, and non-tuberculous and tuberculous salpingitis [1-4]. When the hyperplastic proliferation of the tubal epithelium is florid, the lesion is named "Pseudo-Carcinomatous Hyperplasia" [1-4]. Pseudo-Carcinomatous Hyperplasia $(\mathrm{PCH})$ is rare lesion usually observed incidentally in a slightly enlarged salpinx. However, this entity has also been reported as a pelvic mass which may mimic tubal and ovarian neoplasms, both macroscopically and microscopically [1-4].

On microscopic examination, PCH of the salpinx may be confused with serous carcinoma for the presence of abnormalities in its epithelium, such as a marked mucosal proliferation with a cribriform pattern, nuclear overcrowding, associated with loss of polarity, finely granular chromatin, prominent nucleoli and occasional mitotic figures (Figure 1). However, serous carcinoma of the salpinx may be differentiated from PCH because of other clinical and microscopic findings. Usually, tubal serous carcinoma affects mostly post-menopausal patients. Additionally, tubal PCH can be observed in association with marked chronic inflammation and hyper-estrogenic states. Histologically, tubal PCH can be differentiated from serous carcinoma because this malignancy is characterized by solid epithelial proliferation, prominent nuclear atypia, numerous mitotic figures (Figure 2), and evident invasion of the tubal wall [3]. 


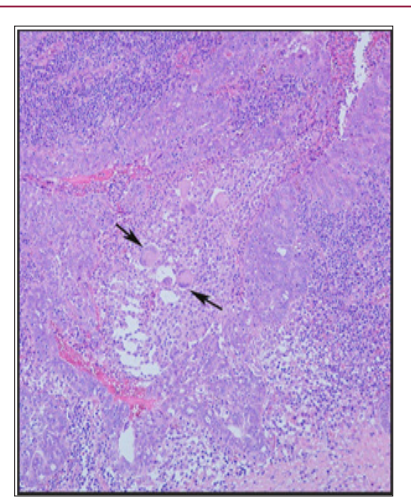

Figure 1: Pseudo-Carcinomatous Hyperplasia of salpinx with marked mucosal proliferation. Note cribriform pattern, nuclear overcrowding and inflammation with multinucleated giant cell (H/Ex100, arrows multinucleated giant cells).

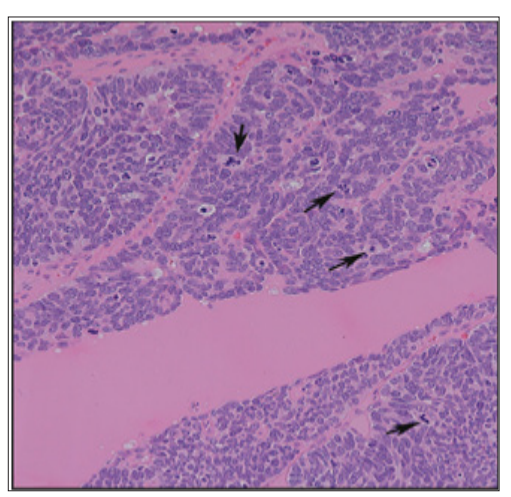

Figure 2: Example of Serous carcinoma of salpinx with solid epithelial proliferation, prominent nuclear atypia, numerous mitotic figures $(\mathrm{H} / \mathrm{E} \times 200$, arrows mitotic figures).

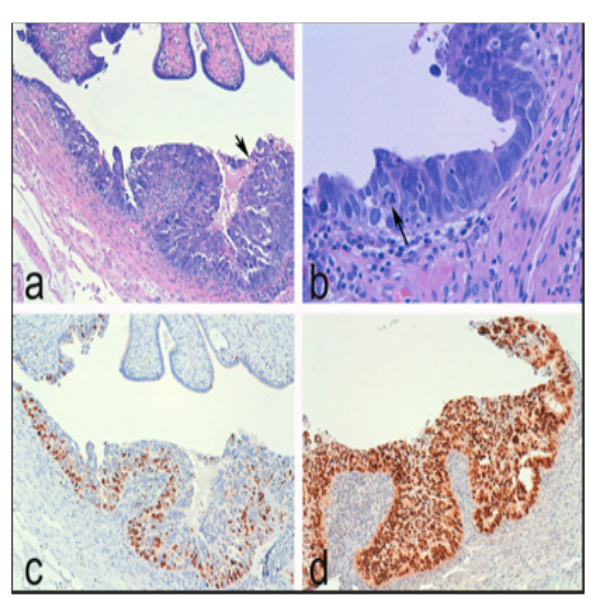

Figure 3: Example of serous tubal intra-epithelial carcinoma (STIC). Note marked nuclear atypia, loss of cell polarity, marked epithelial stratification, small epithelial cell clusters detached from the tubal surface (a: H/E $\mathrm{x}$ 100) the presence of atypical mitosis (b: H/E $x 400$, arrow mitosis), high Ki-67 (c: x 100) and strong nuclear positivity for p53 (c: x200).
Another lesion that could be mistaken for a tubal malignancy is Papillary Syncytial Metaplasia (PSM) of the fallopian tube [5]. This entity can be observed in a salpinx with endometriosis and, like papillary Syncytial Metaplasia of the endometrium, represents a reactive process, due to endometrial breakdown [6-8]. In the salpinx, this lesion may resemble a STIC. However, STIC is characterized by marked nuclear atypia, loss of cell polarity, marked epithelial stratification and the presence of small epithelial cell clusters detached from the tubal surface (Figure 3a) and mitotic figures (Figure 3b). Unlike STIC, PSM presents ciliated cells and acute inflammation, an absence of marked cytologic atypia, and any mitotic activity. Moreover, an immunohistochemical study represents a useful ancillary technique that can differentiate PSM from STIC, revealing high Ki67 index (Figure 3c) and diffuse strong nuclear positivity for p53 in STIC (Figure 3d). However, completely absent p53 staining may be observed in cases of null mutation type of 553 gene.

Transitional cell metaplasia of fallopian tube fimbriae represents other mimic of tubal intraepithelial carcinoma; it was first described by Egan and Russell [9] and had been found incidentally in salpingo-oophorectomy specimens from women with BRCA germline mutations [10]. These mutations, observed in hereditary breast/ovary carcinoma, confer increased risk to develop ovarian, fallopian tube and peritoneal carcinoma. This risk can be reduced by prophylactic salpingo-oophorectomy [10-13]. The differential diagnosis of transitional cell metaplasia of fallopian tube fimbriae and intraepithelial carcinoma may be made because of presence of normal nucleus: cytoplasm ratios, lack of nuclear atypia, presence of nuclear grooves, lack of mitoses (Figure 4a). On immunohistochemical analysis transitional cell metaplasia of fallopian tube fimbriae is negative to p53 and positive to p63 (Figure 4b).

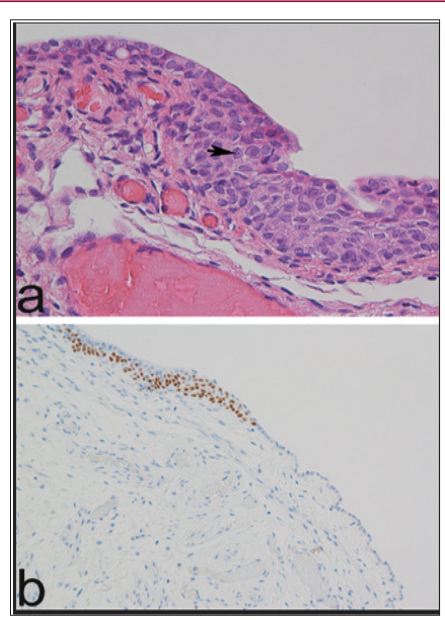

Figure 4: Example of transitional cell metaplasia of fallopian tube fimbriae. Note normal nucleus: cytoplasm ratios, lack of nuclear atypia, presence of nuclear grooves, lack of mitoses (a: H/E x 400, arrows nuclear groove) and nuclear positivity to $\mathrm{p} 63$ (b: $\mathrm{x} 200)$.

\section{Conclusion}

In conclusion, in the salpinx many benign lesions can mimic serous intraepithelial or invasive carcinoma, and here it was 
demonstrated that both morphologic clinical data and immunohistochemical features are useful for an accurate differential pathological diagnosis. Moreover, it is very important the recognition of occult early tubal carcinoma confined to the fimbrial epithelium to prevent the development of ovarian, fallopian tube, and peritoneal carcinoma [10-13]. As well as, it is necessary to keep in mind that uterine serous carcinomas may metastasize to the fallopian tube simulating STIC; thus, its recognition also is useful to made a correct staging of primary serous uterine carcinoma [14].

\section{References}

1. Cheung AN, Young RH, Scully RE (1994) Pseudocarcinomatous hyperplasia of the fallopian tube associated with salpingitis: A report of 14 cases. Am J Surg Pathol 18: 1125-1130.

2. Gupta S, Singh P, Bala J, Mushtaq D, Goyal A (2012) Pseudocarcinomatous hyperplasia of the Fallopian tubes which was associated with female genital tract tuberculosis, histologically mimicking tubal adenocarcinoma: a diagnostic challenge. J Clin Diagn Res 6(8): 14191421.

3. Lee NK, Choi KU, Han GJ, Kwon BS, Song YJ, et al. (2016) Pseudocarcinomatous hyperplasia of the fallopian tube mimicking tubal cancer: a radiological and pathological diagnostic challenge. J Ovarian Res 9(1): 79.

4. Sharma R, Bhartiya V (2017) Giant tuberculous pseudocarcinomatous hyperplasia of fallopian tube mimicking ovarian neoplasm. A challenging diagnosis. Int J Diagn Imaging 4(2): 39-42

5. Kaur H, Levinsky E, Colgan TJ (2013) Papillary Syncytial Metaplsia of fallopian tube endometriosis: a potential pitfall in the diagnosis of serous tubal intraepithelial carcinoma. Arch Pathol Lab Med 137: 126-129.

6. McCluggage WG (2003) Metaplasias in the female genital tract. In: Lowe D, Underwood J (Eds.) Recent advances in histopathology. Royal Society of Medicine Press Ltd, Vol 20 UK.
7. Hendrickson MR, Kempson RL (1980) Endometrial epithelial metaplasias: proliferations frequently misdiagnosed as adenocarcinoma. Report of 89 cases and proposed classification. Am J Surg Pathol 4(6): 525-542.

8. Nicolae A, Preda O, Nogales FF (2011) Endometrial metaplasias and reactive changes: a spectrum of altered differentiation. J Clin Pathol 64: 97-106.

9. Egan AJ, Russell P (1996) Transitional (urothelial) cell metaplasia of the fallopian tube mucosa: morphological assessment of three cases. Int J Gynecol Pathol 15: 72-76.

10. Rabban JT, Crawford B, Chen LM, Powell CB, Zaloudek CJ (2009) Transitional cell metaplasia of fallopian tube fimbriae: a potential mimic of early tubal carcinoma in risk reduction salpingo-oophorectomies from women With BRCA mutations. Am J Surg Pathol 33(1): 111-119.

11. Kauff ND, Barakat RR (2007) Risk-reducing salpingo-oophorectomy in patients with germline mutations in BRCA1 or BRCA2. J Clin Oncol 25(10): 2921-2927.

12. Piek JM, van Diest PJ, Zweemer RP, Jansen JW, Poort-Keesom RJ, et al. (2001) Dysplastic changes in prophylactically removed Fallopian tubes of women predisposed to developing ovarian cancer. J Pathol 195(4): 451-456.

13. Paley PJ, Swisher EM, Garcia RL, Agoff SN, Greer BE, et al. (2001) Occult cancer of the fallopian tube in BRCA-1 germline mutation carriers at prophylactic oophorectomy: a case for recommending hysterectomy at surgical prophylaxis. Gynecol Oncol 80(2): 176-180.

14. Kommoss F, Faruqi A, Gilks CB, Lamshang Leen S, Singh N, et al. (2017) Uterine Serous Carcinomas Frequently Metastasize to the Fallopian Tube and Can Mimic Serous Tubal Intraepithelial Carcinoma. Am J Surg Pathol 41(2): 161-170.



